\title{
RECONHECIMENTO DE ALVOS AÉREOS SUSPEITOS VISANDO A PROTEÇÃO DE PONTOS DE INTERESSE EM ÁREAS EXTENSAS
}

\author{
Douglas de Oliveira Marques \\ EMBRAER S.A. \\ Av. Brigadeiro Faria Lima, 2170 - São José dos Campos, SP - 12227-901 \\ douglas.oliveira@embraer.com.br \\ José Fernando Basso Brancalion \\ EMBRAER S.A. \\ Av. Brigadeiro Faria Lima, 2170 - São José dos Campos, SP - 12227-901 \\ jose.brancalion@embraer.com.br \\ Prof. Dr. Karl Heinz Kienitz \\ Instituto Tecnológico de Aeronáutica - ITA \\ Praça Marechal Eduardo Gomes, 50 - - São José dos Campos, SP - 12228-900 \\ kienitz@ita.br
}

\section{Resumo}

Este trabalho trata do reconhecimento (compreendendo a identificação e classificação automática) de alvos com comportamentos hostis em relação a um ponto de interesse, baseado em redes bayesianas, sendo uma ferramenta de suporte à tomada de decisão com potencial significativo de aplicação na vigilância de grandes áreas.

Palavras-chave: Avaliação de ameaças; Redes Bayesianas; Classificação Automática.

\begin{abstract}
This work is related to the reconnaissance (including the automatic identification and classification) of targets with hostile behaviors in relation to one point of interest, based on Bayesian Networks, being a decision making support tool with great potential applicable in the surveillance of large areas.
\end{abstract}

Keywords: Threat Assessment; Bayesian Networks; Automatic Classification. 


\section{INTRODUÇÃO}

Este trabalho trata do reconhecimento (compreendendo a identificação e classificação automática) de alvos com comportamentos hostis em relação a um ponto de interesse, baseado em redes bayesianas dinâmicas, sendo uma ferramenta de suporte à tomada de decisão com potencial significativo de aplicação na vigilância de grandes áreas.

A vigilância de grandes áreas é um desafio encontrado pelas Forças Armadas. Necessita-se de um conjunto significativo de equipamentos móveis (por exemplo, aeronaves, helicópteros, veículos aéreos não tripulados, navios) e fixos (por exemplo, radares terrestres) para vigiar áreas geográficas de grande abrangência a fim de identificar, avaliar e rastrear os agentes presentes neste ambiente [1].

A limitação dos recursos existentes para vigiar e neutralizar ameaças em uma região tão vasta exige a aplicação de técnicas que permitam avaliar e priorizar aqueles agentes que representam maior perigo para os recursos e entidades presentes no cenário. Devem-se priorizar os recursos e os pontos vitais selecionados e priorizados como pontos ou áreas sensíveis em função de sua importância.

Um exemplo de uma vasta região que exige o monitoramento constante devido ao seu valor estratégico para a nação é a região do "pré-sal". A região da "camada pré-sal" está localizada na bacia sedimentar litorânea brasileira, mais precisamente, entre os litorais do Espírito Santo e Santa Catarina em uma extensão de cerca de 800 quilômetros abrangendo a região de três bacias sedimentares: as bacias do Espírito Santo, Campos e Santos. A área de ocorrência conhecida destes reservatórios é de 149 mil km², apresentada da Figura 1.

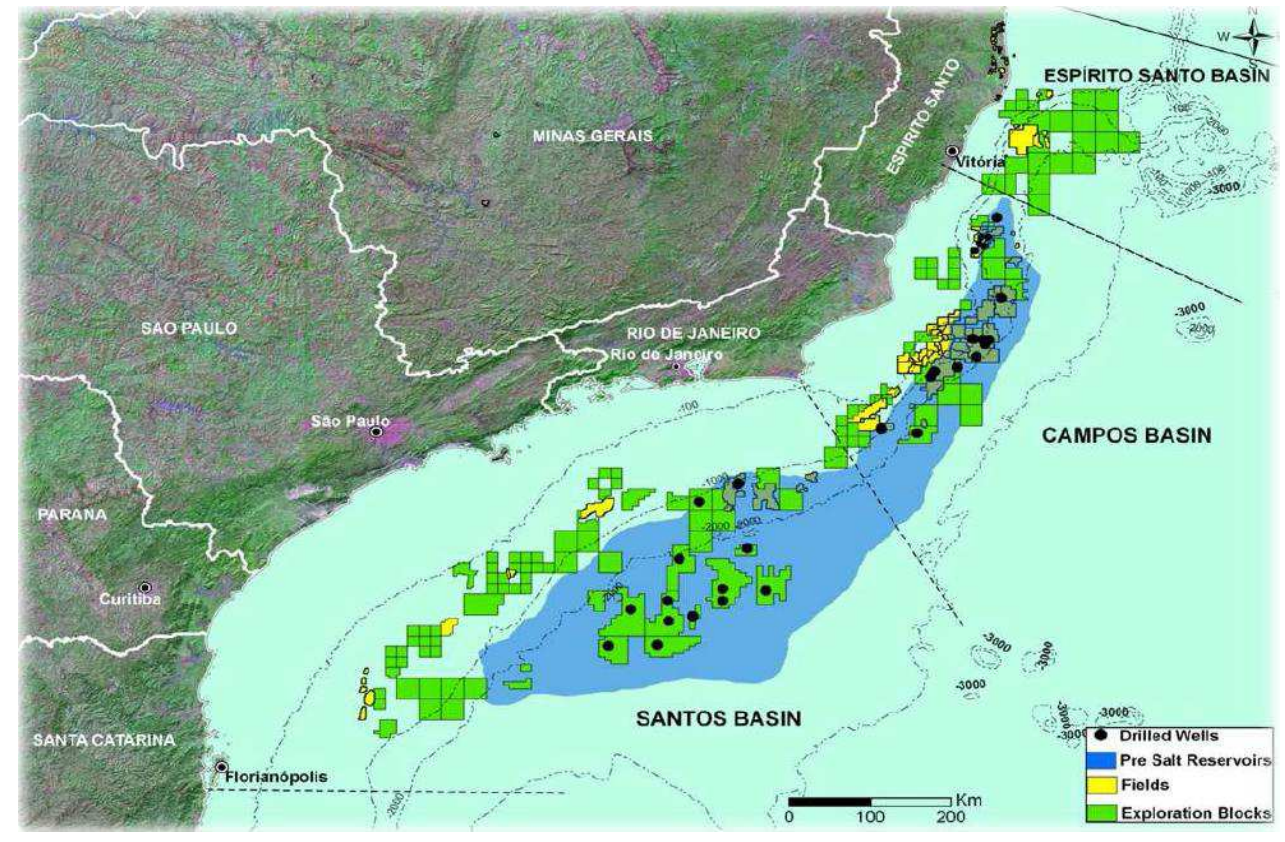

Figura 1: Região do pré-sal. [2]

No contexto militar as informações referem-se aos agentes de interesse militar tais como aviões, helicópteros, mísseis, navios, carros, balões, etc. Os dados utilizados nos sistemas de fusão de dados são gerados por diversos tipos de sensores. Os sistemas táticos existentes carecem de uma fusão de informações de mais alto nível onde sejam integradas informações dos sensores, do ambiente, do contexto geopolítico, do contexto operacional e das doutrinas de forma a otimizar o processo de auxílio à tomada de decisão em períodos exíguos de tempo [3]. 
A disponibilização de informações sobre o teatro de interesse para os tomadores de decisão com exatidão requer que os dados e as informações de diversas fontes sejam coletadas, processadas (analisadas quando necessário), transportadas, agrupadas, colocadas no contexto apropriado e apresentadas de modo a facilitar inferências rápidas e precisas.

Um Sistema de Fusão de Dados de Alto Nível transforma os dados de entrada em informações de Inteligência. Em [4], Waltz descreve esta transformação de dados em informação, e de informação em conhecimento. O nível de cognição é acrescentado para demonstrar o objetivo final de antecipar os eventos contrários. A Figura 1 apresenta o nível cognitivo como o nível mais alto da cadeia de exploração de dados.
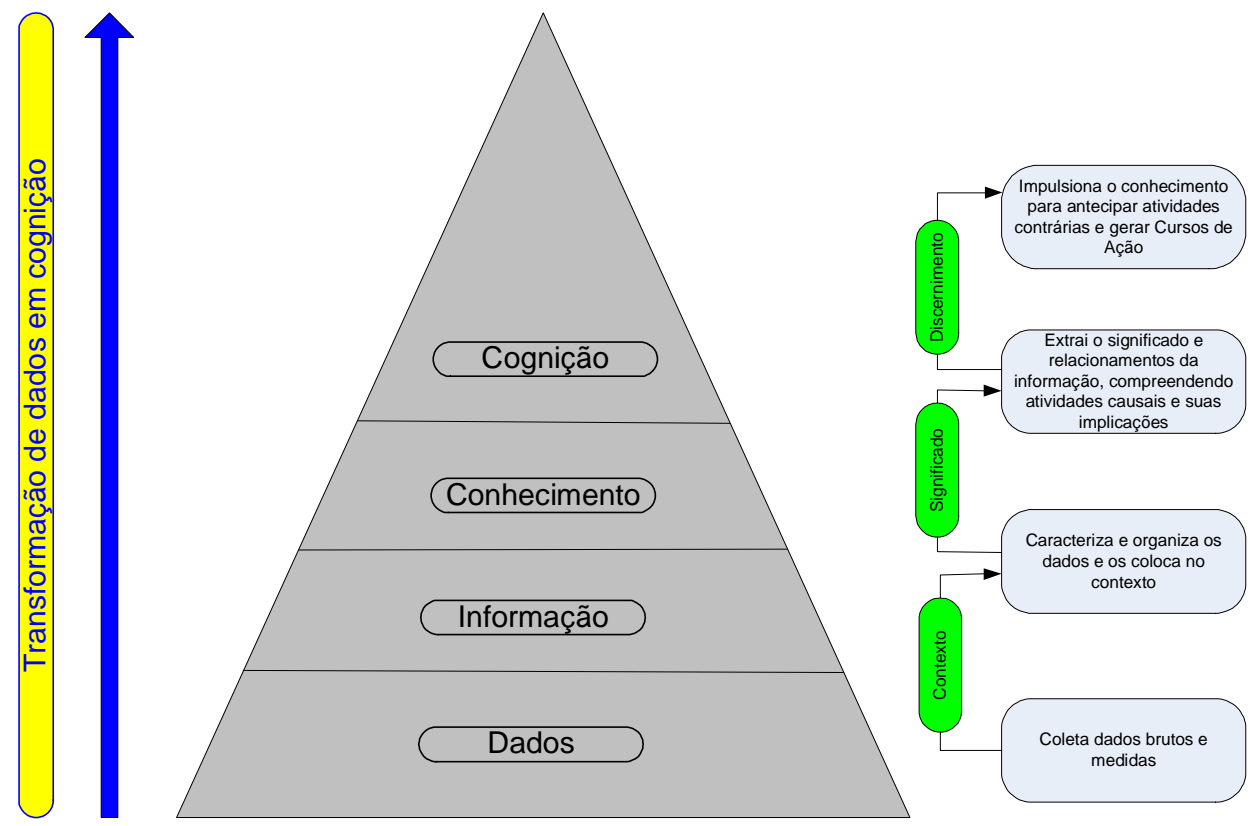

Figura 1: Cadeia de processamento: dos dados à cognição.

O sistema de fusão de dados de alto nível fornece aos tomadores de decisão o conhecimento essencial para que possam avaliar onde os problemas podem ocorrer ou onde eles já existem, qual o tipo de problema e qual o impacto destes problemas. A partir da avaliação destas questões é possível construir uma avaliação situacional da situação corrente e prever seus impactos no futuro. Pode-se então definir a avaliação situacional como um processo que avalia os elementos e as situações existentes e seus relacionamentos a fim de fornecer como resultado a Consciência Situacional para o tomador de decisão.

A fusão de dados de alto nível deve incluir o fornecimento ou aprimoramento da Consciência Situacional, composta pelas funções de estimativa e predição de relacionamentos, e a Avaliação de Impactos que inclui a estimativa e a predição dos efeitos obtidos de acordo com as ações executadas. [5]

O objetivo da Consciência Situacional é determinar se alguma das atividades que estão sendo executadas tem algum impacto nos meios e recursos de interesse, ou se terão algum impacto futuro [6]. Na avaliação de impacto pode-se estimar o dano associado a um determinado meio e a partir dessa análise traçar estratégias para minimizar ou evitar tais danos.

A avaliação situacional e a avaliação de impacto são processos dinâmicos, que refletem as mudanças no cenário ao longo do tempo, fornecendo aos tomadores de decisão os possíveis estados do ambiente associados com uma plausibilidade.

A análise de avaliação de ameaças deve considerar os seguintes fatores: capacidade, oportunidade e intenção. Alguns elementos contribuintes para cada fator apresentado são 
listados a seguir:

- Capacidade: treinamento, habilidades, conhecimento, recursos, armas, modos de operação, organização.

- Oportunidade: acessos aos alvos, modo de operação, localização, vulnerabilidades.

- Intenção: motivação, comportamentos anômalos (desvios), atividades relacionadas a uma cadeia de eventos.

Diversos trabalhos relacionados à avaliação de ameaças visando o aprimoramento da Consciência Situacional em sistemas de defesa aérea têm sido desenvolvidos, utilizando-se de diferentes técnicas como sistemas baseados em regras [7] ou lógica fuzzy [8].

Este trabalho propõe um sistema de identificação e classificação automática de alvos com comportamentos hostis em relação a um ponto de interesse, baseado em redes bayesianas dinâmicas, sendo uma ferramenta de suporte à tomada de decisão. Outros trabalhos similares podem ser encontrados em [9] ou [10]. O comportamento das aeronaves é monitorado ao longo da sua trajetória e o sistema automaticamente classifica e identifica as aeronaves que possivelmente representam uma ameaça à um ponto de interesse em solo.

A Seção 2 apresenta a descrição dos sistemas de fusão de dados de alto nível e sua distinção em relação aos sistemas de fusão de dados de baixo nível. A Seção 3 apresenta a descrição teórica sobre redes Bayesianas, principal ferramenta teórica e de processamento utilizada na proposta desse trabalho. A Seção 4 apresenta o gerador de trajetórias utilizado nos experimentos. A Seção 5 apresenta os resultados experimentais obtidos e a Seção 6 finaliza o artigo com as conclusões e propostas para trabalhos futuros.

\section{SISTEMAS DE FUSÃO DE DADOS DE ALTO NÍVEL}

As motivações tradicionais da comunidade de fusão de dados estão relacionadas à fusão de dados de sensores, onde as fontes de dados são constituídas por sensores e os itens de interesse no ambiente são objetos móveis, cada um representado por um vetor de estado. Uma definição mais abrangente reflete o crescente interesse em estender a fusão de dados sensoriais para a fusão de dados de alto nível, onde os aspectos de interesse do ambiente não se restringem apenas aos objetos [11]. A fusão de informações de alto nível é a capacidade de um sistema de fusão, através de conhecimento, experiência e entendimento de produzir a consciência situacional e identificar relacionamentos complexos, obter inferências sobre eventos passados e futuros, analisar a utilidade dos resultados a fim de atingir os objetivos desejados pelo sistema para cada nível. Estes processos devem trabalhar em cenários onde os valores estão em constante mudança, que é uma característica de sistemas de tempo real.

A distinção entre fusão de dados de baixo nível e fusão de dados de alto nível foi realizada primeiramente por Waltz e Llinas [12]. A Figura 3 apresenta os processamentos e saídas de um sistema de fusão de dados. 


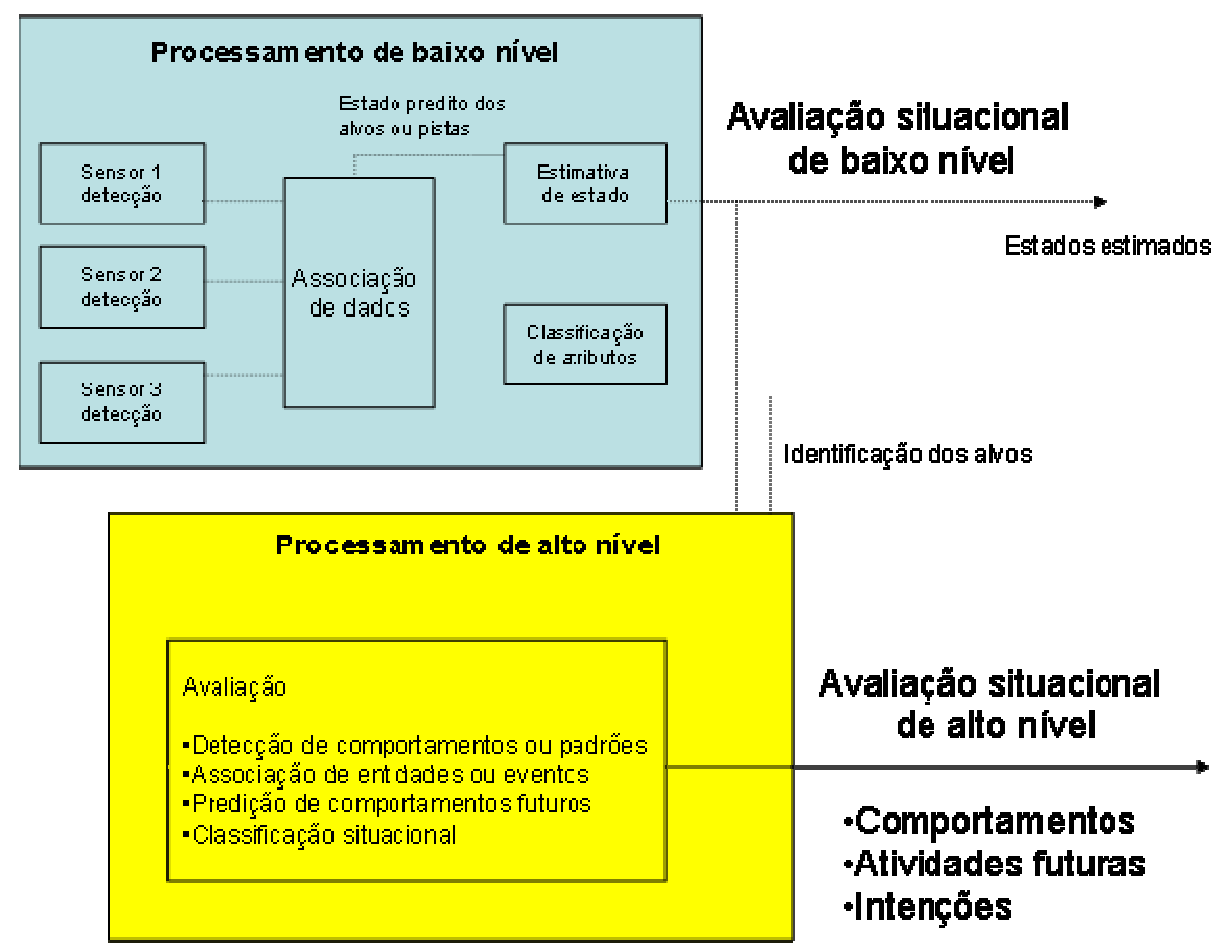

Figura 3: Funções de um sistema de fusão de dados.

\section{REDES BAYESIANAS}

A teoria de probabilidades Bayesiana é uma ferramenta muito poderosa para a construção de modelos de fenômenos envolvendo incertezas. As probabilidades expressam graus de plausibilidade ou probabilidade variando de eventos com alto grau de certeza até eventos onde a probabilidade de ocorrência é impossível. Os modelos Bayesianos podem combinar o conhecimento tácito dos especialistas com dados observados e podem ser redefinidos ao longo do tempo tanto pelo aprendizado quanto pela observação [13].

As redes Bayesianas permitem a realização da inferência Bayesiana, que é o cálculo do impacto de valores observados de um subconjunto de variáveis modeladas na distribuição de probabilidade das outras variáveis. A atualização Bayesiana, também conhecida de atualização de crenças, é baseada nos parâmetros numéricos capturados no modelo. Todos os algoritmos para atualização Bayesiana são baseados no teorema proposto por Rev. Thomas Bayes, conhecido como Teorema de Bayes.

Uma rede Bayesiana caracteriza um domínio de problema que consiste em um conjunto de variáveis aleatórias $U=\{X 1, \ldots, X n\}$. Estas variáveis estão em uma rede Bayesiana representada como um conjunto de nós (vértices) $\mathrm{V}$ em um grafo dirigido sem ciclos $\mathrm{G}=(\mathrm{V}, \mathrm{E})$, onde o conjunto de vértices $\mathbf{E} \subseteq \mathbf{V} \times \mathbf{V}$ especifica relações de dependência e independência condicional entre as variáveis que fazem parte do domínio. A Figura 4 ilustra um exemplo de uma rede Bayesiana, representando um sistema de detecção de lançamento de mísseis. 


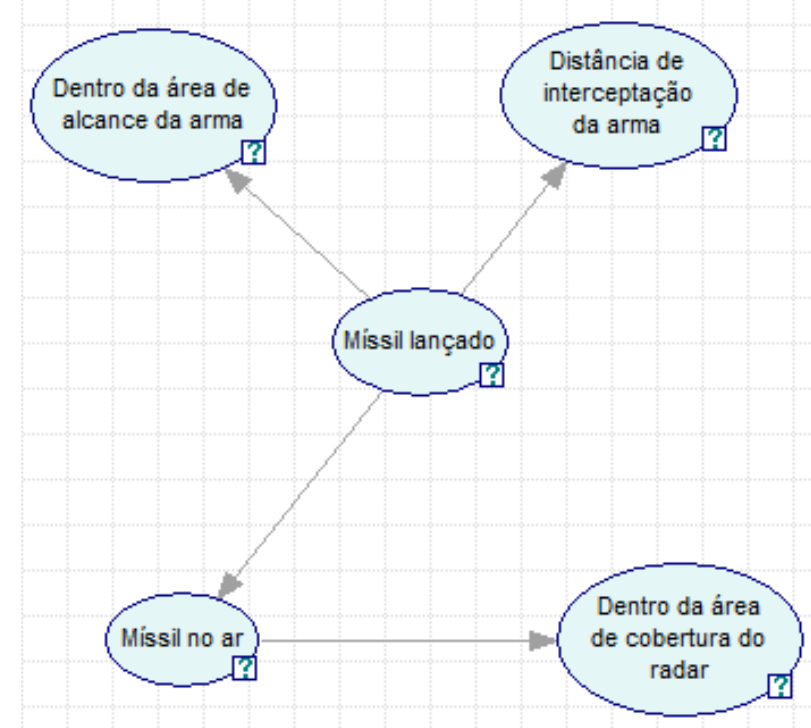

Figura 4: Ilustração de uma rede Bayesiana representando um sistema para detecção de lançamento de mísseis (adaptado de [9]).

Dada o grafo $\mathrm{G}$, a distribuição de probabilidade conjunta $\mathrm{P}$ sobre $\mathrm{U}$ pode ser calculada a partir do conjunto das distribuições de probabilidades locais associadas a cada nó Xi,usando a regra de encadeamento das redes Bayesianas:

$$
P\left(x_{1}, \ldots, x_{n}\right)=\prod_{i=1}^{n} P\left(x_{i} \mid p a_{i}\right)
$$

onde o conjunto das distribuições de probabilidade locais consiste das distribuições no produto da Equação 1 (com $p a_{i}$ refere-se a atribuição de valores do conjunto pai $P A_{i}$ do nó $\mathbf{X}_{\mathbf{i}}$ ). A probabilidade de distribuição conjunta pode ser vista como a função atribuindo um número na faixa $[0,1]$ a cada combinação possível dos estados das variáveis que descrevem o domínio. A força das redes Bayesianas consiste na sua habilidade de representar as distribuições de probabilidade conjuntas em uma maneira compacta, devido à codificação das dependências condicionais entre diferentes variáveis no domínio.

\section{GERADOR DE TRAJETÓRIAS}

Para criar as trajetórias das aeronaves foi desenvolvido um módulo gerador de trajetórias em ambiente Matlab, cuja interface gráfica é apresentada na Figura 5. O módulo permite a criação de trajetórias complexas através da junção de diferentes segmentos de trajetórias, onde é possível selecionar um modelo dinâmico associado a cada segmento. Os modelos dinâmicos existentes no gerador são Velocidade Constante (CS), Curva Coordenada 2-D (2CT) e Curva Variável Planar (PVT). A descrição de cada um destes modelos pode ser encontrada em [14]. Para cada trajetória definem-se a posição inicial em coordenadas de latitude e longitude, as velocidades e acelerações iniciais da aeronave nos eixos x, y, z, a duração e o modelo dinâmico associado a cada segmento da trajetória. 


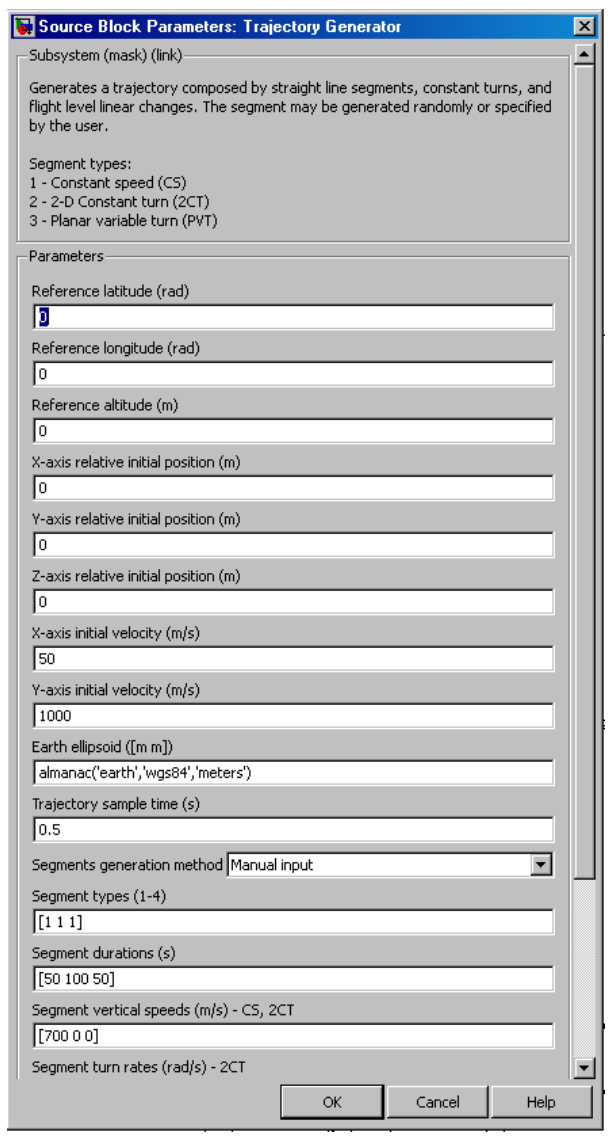

Figura 5: Módulo gerador de trajetórias.

\section{RESULTADOS EXPERIMENTAIS}

O cenário de interesse foi simulado em ambiente Matlab, sendo constituído por duas aeronaves que se deslocam em direção a um ponto de interesse localizado em solo. Neste experimento o gerador de trajetórias produz periodicamente como saída um vetor contendo as informações da posição, velocidade e aceleração das aeronaves. Estas informações servem de entrada para a rede Bayesiana que foi definida no software GeNIe [15], [16].

O classificador identifica dinamicamente o tipo de aeronave, baseado em suas características cinemáticas, sendo possíveis as seguintes classificações: Aeronave de Pequeno Porte, Aeronave Comercial, Aeronave de Alto Desempenho e Outros.

O mecanismo de inferência identifica dinamicamente os riscos associados a cada uma das aeronaves, sendo que o Risco 1 representa o menor grau de ameaça da aeronave em relação ao ponto de interesse e o Risco 5 representa o maior grau de ameaça. $\mathrm{O}$ grau de ameaça está relacionado à maior probabilidade da aeronave realizar uma ação hostil e infringir danos ao ponto de interesse.

A Figura 6 e a Figura 7 representam as trajetórias simuladas para as aeronaves e suas posições e altitudes em relação a um ponto de interesse localizado em solo. A partir deste ponto de interesse são geradas regiões concêntricas, onde cada região representa um grau de ameaça da aeronave em relação ao ponto. As regiões mais distantes representam um grau de ameaça menor que aumenta à medida que se aproximam do ponto de interesse. 


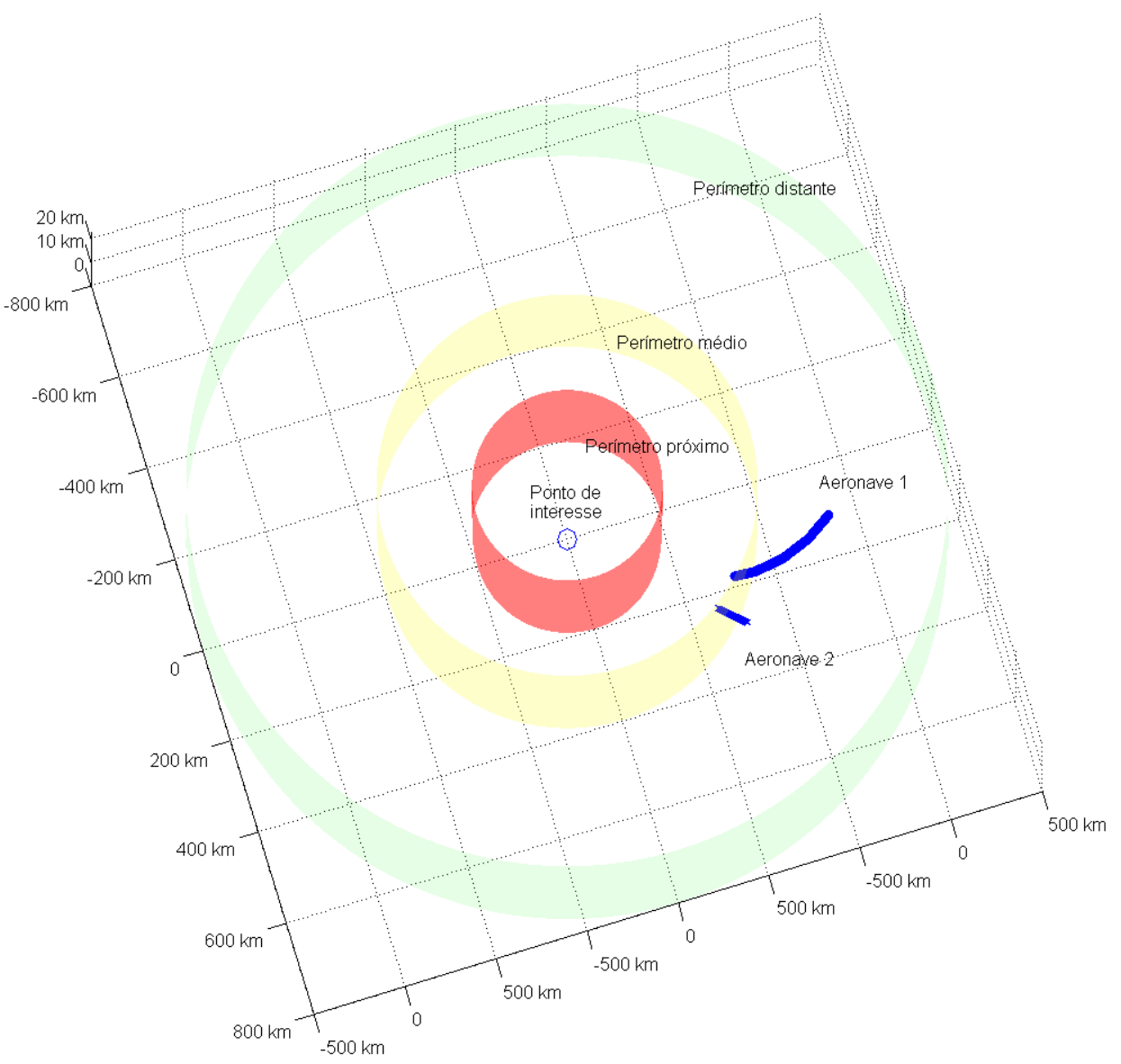

Figura 6: Trajetórias simuladas das aeronaves em torno do ponto de interesse.
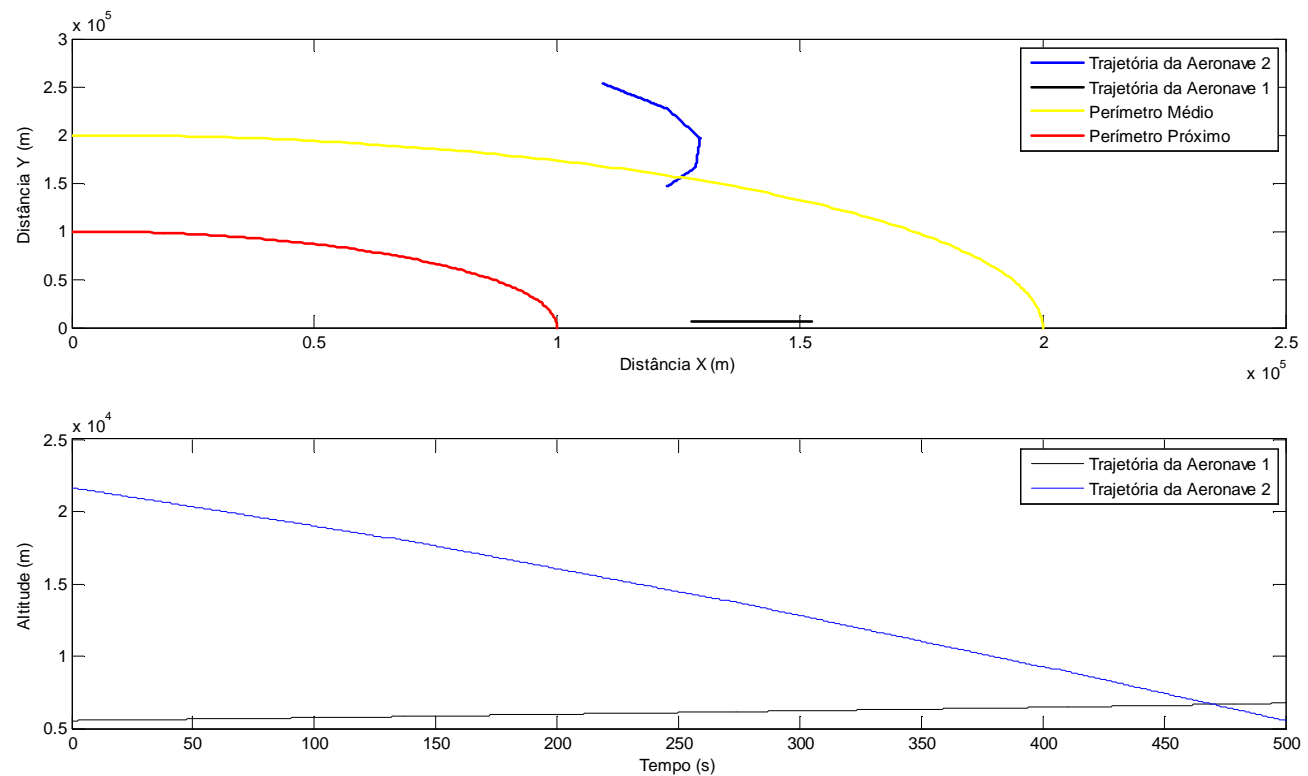

Figura 7: Posições e altitudes das aeronaves em relação ao ponto de interesse, localizado na posição $(0,0)$.

A Figura 8 e a Figura 9 apresentam a rede Bayesiana e sua estrutura, utilizada no sistema de análise de ameaças, onde o grau de ameaça é inferido dinamicamente a partir das 
informações da distância da aeronave (se a aeronave está no perímetro próximo, médio ou distante) em relação ao ponto de interesse, da velocidade e do tipo da aeronave (obtido a partir da estimação das informações cinemáticas da aeronave (velocidade e aceleração) e altitude).

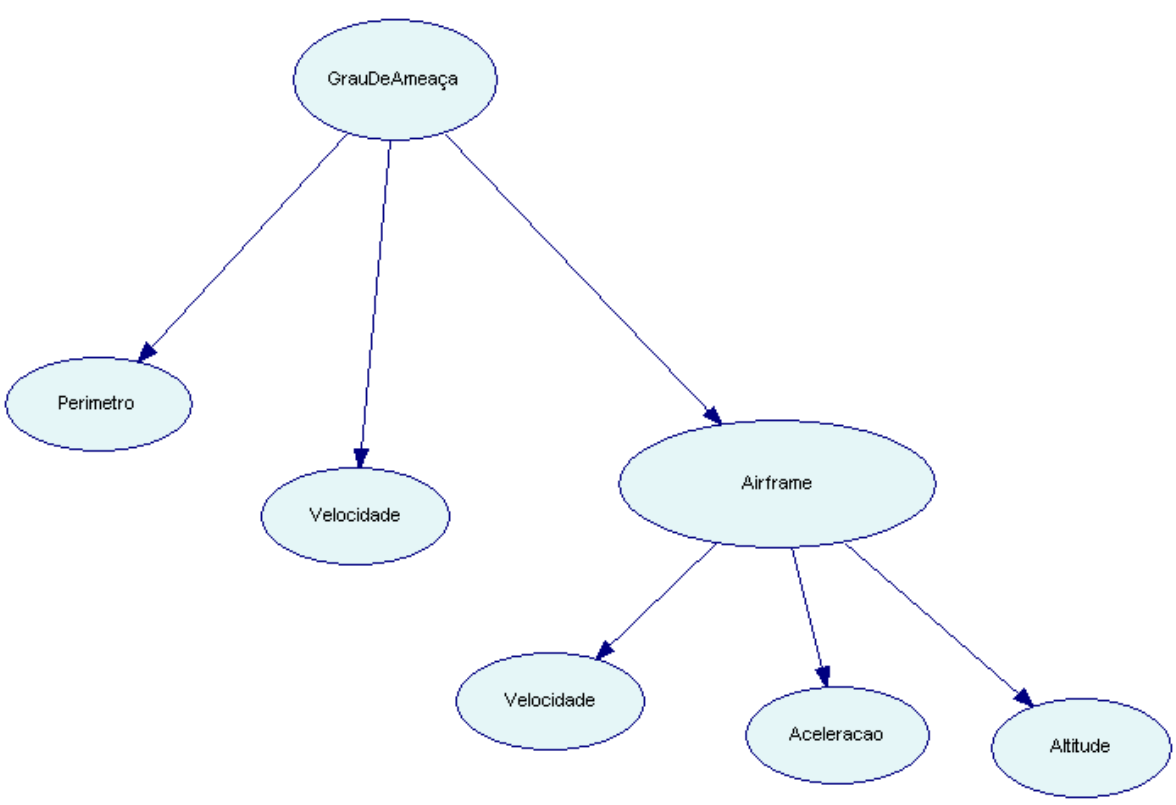

Figura 8: Rede Bayesiana descrevendo a situação de interesse.

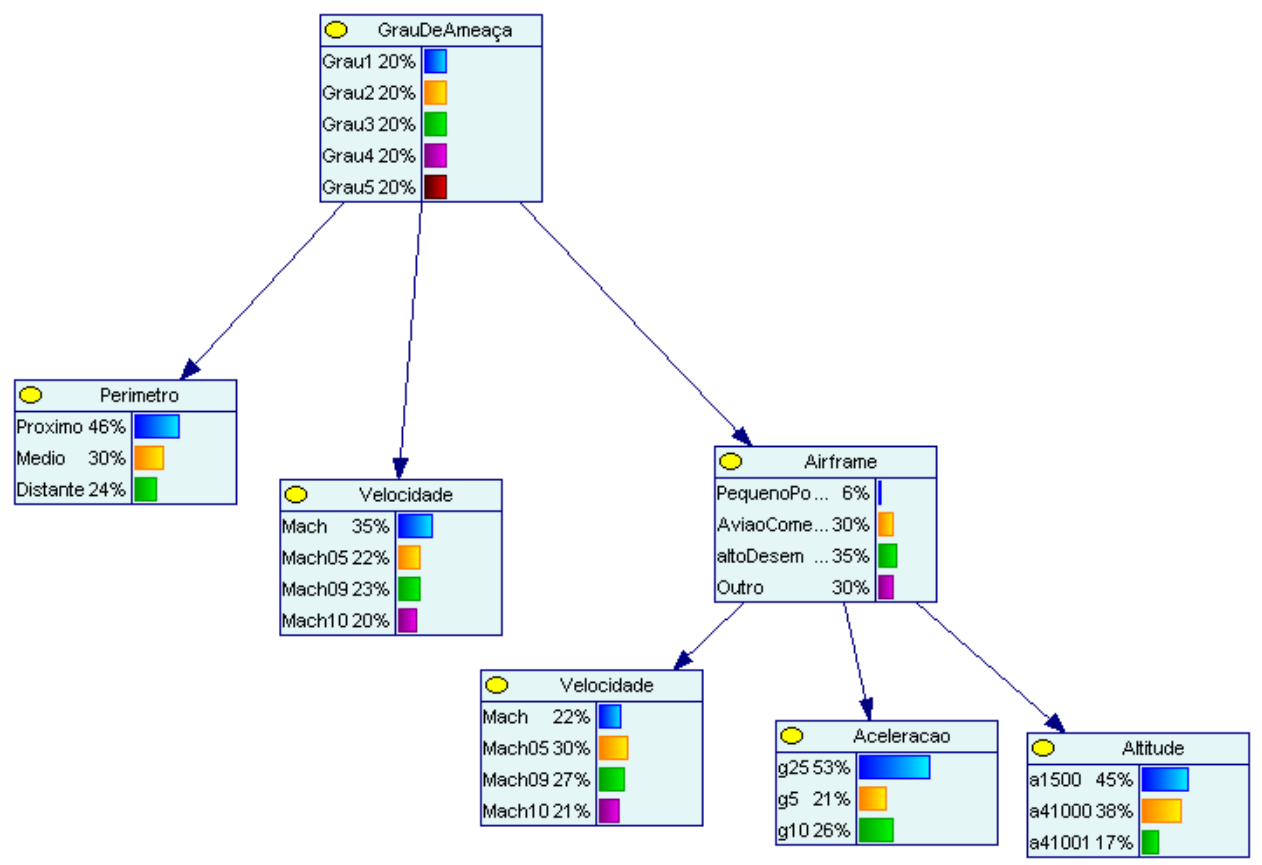

Figura 9: Estrutura da rede Bayesiana utilizada no sistema de análise de ameaças.

A Figura 10 apresenta o ambiente integrado constituído pelo gerador de trajetórias em ambiente Matlab e pela ferramenta GeNIe utilizada para criar a rede Bayesiana. 


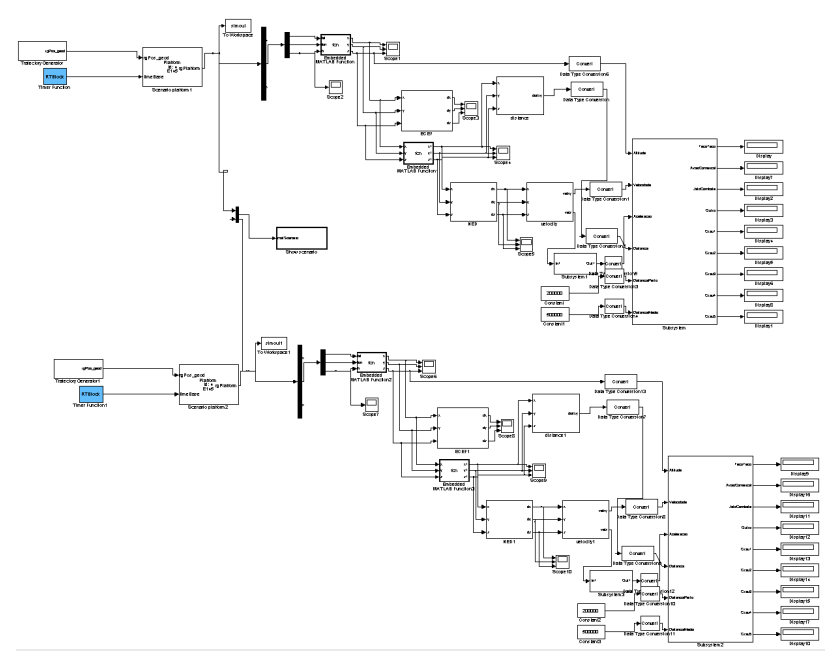

Figura 10: Sistema experimental integrado: gerador de trajetórias criado em ambiente Matlab e classificadores do tipo de aeronave e do nível de ameaça utilizando-se o software GeNIe.

A Figura 11 apresenta a classificação do tipo de aeronave e do grau de ameaça associado à aeronave 1 . Ao longo do tempo verifica-se que as probabilidades relacionadas ao tipo de aeronave se modificam, inicialmente a maior probabilidade estava relacionada à aeronave ser do tipo Avião Comercial, até o momento que o classificador baseando-se na informação de que a velocidade estimada da aeronave era muito alta, aumentou a probabilidade de classificação para aeronave do tipo Alto Desempenho, diminuindo a probabilidade de classificação relacionada à aeronave do tipo Avião Comercial. $\mathrm{O}$ grau de ameaça é calculado ao longo da trajetória da aeronave. Como a aeronave está se aproximando do ponto de interesse, sua altitude está diminuindo e sua classificação de tipo mudou de Aeronave Comercial para Alto Desempenho o grau de ameaça associado também aumentou, diminuindo a probabilidade associada ao grau 2 e aumentando as probabilidades associadas aos graus 4 e 5 .
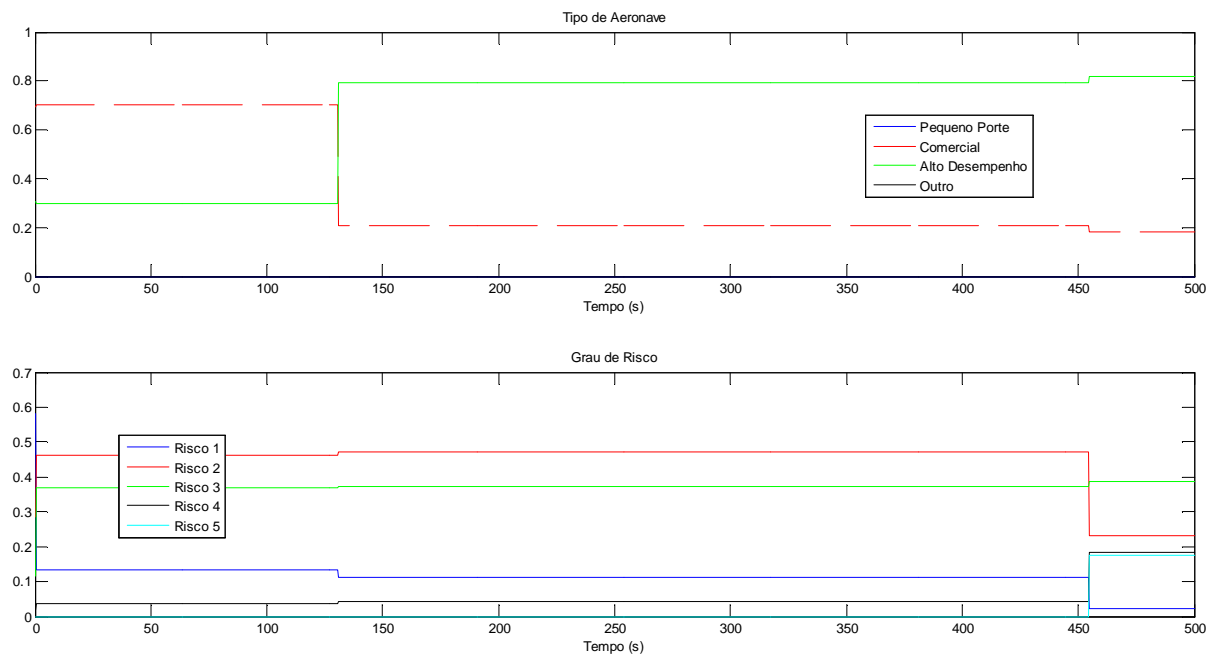

Figura 11: Classificação do tipo da aeronave 1 e do grau de ameaça representado por ela.

A Figura 12 apresenta a classificação do tipo de aeronave e do grau de ameaça associado à aeronave 2 . Ao longo do tempo verifica-se que as probabilidades relacionadas ao tipo de aeronave se modificam, inicialmente a maior probabilidade estava relacionada à aeronave ser do tipo Pequeno Porte, permanecendo até o final da trajetória. O grau de ameaça é calculado ao longo da trajetória da aeronave. Como a aeronave está se afastando do ponto de 
interesse, sua altitude variou muito pouco e sua classificação de tipo permaneceu como aeronave de Pequeno Porte a probabilidade relacionada ao grau de risco 1, correspondente ao menor grau de ameaça, aumentou durante a trajetória.
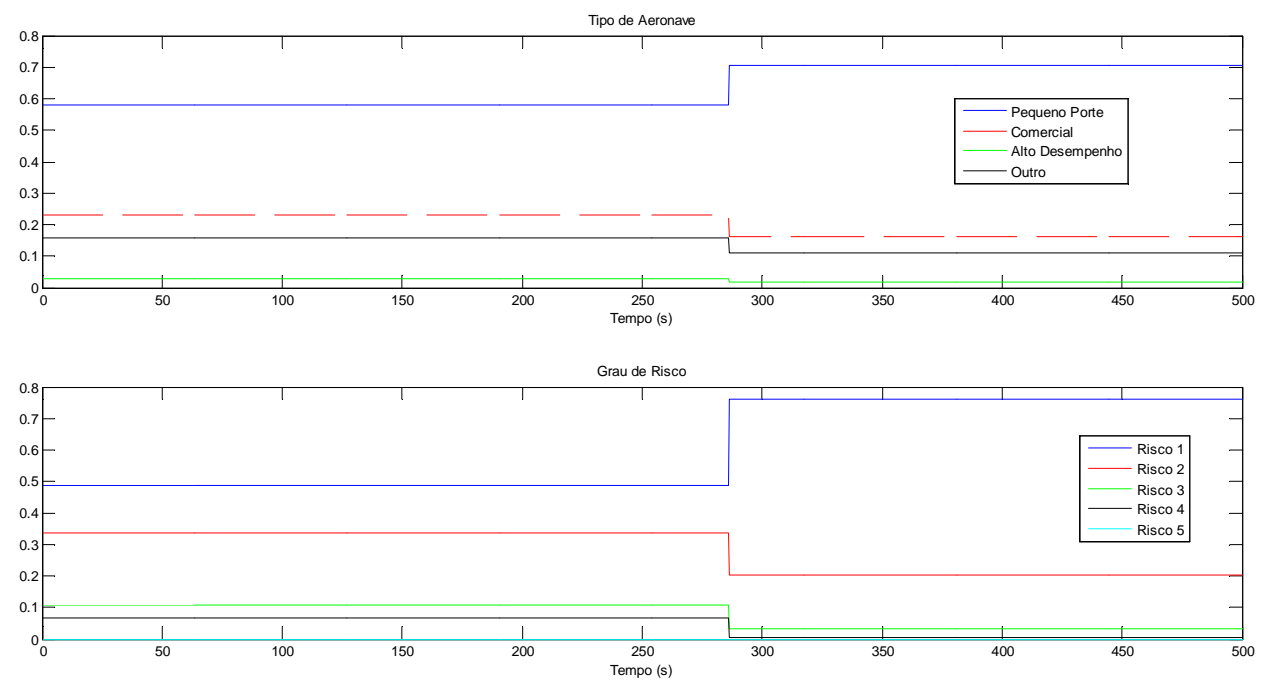

Figura 12: Classificação do tipo da aeronave 2 e do nível de ameaça representado por ela.

\section{CONCLUSÃO}

Neste artigo foram apresentados os conceitos dos sistemas de fusão de dados de baixo nível e de alto nível. Foi descrito um modelo representativo dos processos associados aos sistemas de fusão de dados de alto nível, que busca identificar em um cenário composto por diversos agentes, o mais hostil e que representa maior ameaça a um determinado recurso. Este sistema constitui-se em um elemento muito importante para os sistemas de defesa, dado que a área a ser protegida é muito grande e os recursos são escassos, devendo-se, portanto avaliar qual a melhor maneira de ser utilizar os meios disponíveis para a defesa. Como extensão deste trabalho propõe-se a utilização de um planejador automático integrado ao sistema de análise de ameaças, visando gerar planos automáticos de combate às ameaças identificadas. Outra sugestão seria a inclusão de outros nós na rede Bayesiana representando outras informações a respeito dos alvos provenientes de outras fontes de informação.

\section{REFERÊNCIAS BIBLIOGRÁFICAS}

[1] Brancalion, J.F.B.; Kienitz, K.H. Fusão de Dados de Alto Nível: Aplicações e Desafios. Anais do XIII SIGE, Setembro, 2011.

[2] http://fatosedados.blogspetrobras.com.br/2009/07/28/pre-sal-sucesso-garantido/. Acesso em abril de 2014.

[3] Gomes, J.G.C. Fusão de dados multi-nível em ambientes de monitoração contínua de sistemas táticos navais utilizando múltiplas lógicas. Tese de doutorado. UFRJ, 2008.

[4] Waltz, E.L. Information Understanding: Integrating Data Fusion and Data Mining Processes. Proceedings of the 1998 IEEE International Symposium on Circuits and Systems (ISACAS '98), vol. 6, p. 553-556, 1998. 
[5] Toth, G.; Kokar, M.M.; Wallenius, K.; Laskey, K.B.; Sudit, M.; Hultner, M.; Kessler, O. Higher-Level Information Fusion: Challenges to the Academic Community. $11^{\text {th }}$ International Conference on Information Fusion. Colônia, Alemanha, 2008.

[6] Salerno, J.J.; Tadda, G.P. Ranking Activities Based on Their Impact and Threat. $12^{\text {th }}$ International Conference on Information Fusion. Seatle, EUA, Julho, 2009.

[7] Liebhaber, M.J.; Feher, B. Air Threat Assessment Research, Model and Display Guidelines. Proceedings of the 2002 Command and Control Research and Technology Symposium, 2002.

[8] Johansson, F.; Falkman, G. A. Comparison Between Two Approaches to Threat Evaluation in an Air Defense Scenario. Proceedings of the $5^{\text {th }}$ International Conference on Modeling Decisions for Artificial Intelligence. Sadabell, Espanha, 2006.

[9] Dahlbom, A.; Nordlund, P. Detection of Hostile Aircraft Behaviors Using Dynamic Bayesian Networks. 16 ${ }^{\text {th }}$ International Conference on Information Fusion. Istambul, Turquia, Julho, 2013.

[10] Louvieris P.; Gregoriades A.; Garn W. Assessing critical success factors for military decision support. Expert Systems with Applications. n. 37, p. 8229-8241, 2010.

[11] Lambert, D.A. A Blueprint for High-Level Fusion Systems. Information Fusion, vol. 10, p. 6-24, Janeiro, 2009.

[12] Waltz, E.; Llinas, J. Multisensor Data Fusion. Artech House, Norwood, MA, 1990.

[13] Laske K.; Alghamdi G.; Wang X. Detecting Threatening Behavior Using Bayesian Networks. $13^{\text {th }}$ Conference on Behavior Representation in Modeling and Simulation EUA, Maio, 2004.

[14] Li X.R.; Jilkov V.P. Survey of Maneuvering Target Tracking Part I: Dynamic Models. IEEE Transactions on Aerospace and Electronic Systems. vol. 39, n. 4, p. 1333-1364, Outubro, 2003.

[15] Druzdzel, M.J. SMILE: Structural Modeling, Inference, and Learning Engine and GeNIe: a development environment for graphical decision-theoretic models. Proceedings of the AAAI/IAAI. 1999. p. 902-903.

[16] DSI, "GeNIe and SMILE", Decision Systems laboratory, Universidade de Pittsburgh, PA, EUA, 2013. 